Proceedings of the 2012 Winter Simulation Conference

C. Laroque, J. Himmelspach, R. Pasupathy, O. Rose, and A. M. Uhrmacher, eds.

\title{
MODELING SOCIAL GROUPS IN CROWDS USING COMMON GROUND THEORY
}

\author{
Seung In Park \\ Francis Quek \\ Yong Cao \\ Virginia Tech \\ Blacksburg, VA 24061, USA
}

\begin{abstract}
Social interaction and group coordination are important factors in the simulation of human crowd behavior. To date, few simulation methods have been informed by models of human group behavior from the social science studies. In this paper we advance a computational model informed by Common Ground (CG) Theory that both inherits the social realism provided by the CG model and is computationally tractable for a large number of groups and individuals. The task of navigation in a group is viewed as performing a joint activity among agents, which requires effective coordination among group members. Our model includes both macro and micro coordination, addressing the joint plans, and the actions for coordination respectively. These coordination activities and plans inform the high-level route and walking strategies of the agents. We demonstrate a series of studies to show the qualitative and quantitative differences in simulation results with and without incorporation of the CG model.
\end{abstract}

\section{INTRODUCTION}

In the simulation of human crowd behavior including evacuation planning, transportation management, and safety engineering in architecture design, the development of pedestrian model for higher behavior fidelity is an important issue. Simulations should exhibit human behaviors for navigation, pedestrian decision-making, collision avoidance, and social behaviors such as grouping and crowding. To date, few simulation models have been informed by models of human group behavior from the social sciences. In this paper, we will advance a model that is founded on sound social science that we have formulated into a computable framework.

One common approach to the simulation of mass flow involves the application of fluid dynamics (Hughes 2002; Narain, Golas, Curtis, and Lin 2009). To the degree that humans are physical entities, and exhibit some particle-like behavior in crowds, fluid dynamics-based models are able to model such behavior. However, humans are autonomous deciders, and these decisions result in complexity that simple fluid dynamics modeling cannot address. Agent-based models allow for each pedestrian to be modeled as an autonomous entity capable of perceiving its environment and interacting with other agents. Several approaches to pedestrian simulation have adopted an agent-based modeling to incorporate the impact of cognitive, psychological, emotional, and cultural factors in the simulation of virtual agents (Rao and Georgeff 1995; Hoogendoorn and Bovy 2004; Antonini, Bierlaire, and Weber 2006; Endrass, André, Rehm, Lipi, and Nakano 2011), however these have focused on the behavior of individual agents and not the overall coordination among agents in groups, and do not scale to a large number of groups.

Social science field studies inform us that crowds are not made up of a mass of isolated individuals. Up to $70 \%$ of observed pedestrians are walking in groups (James 1953; Coleman and James 1961), and hence one component of the complexity of crowd dynamics emerges from the presence of various patterns of social interactions within groups that make up the crowd. Therefore pedestrian interaction should be modeled by taking a perspective of mindful agents as group members. Some attempts have been made 
to reproduce group dynamics such as cohesive movements and spatial group structures by manipulating agents only at a reactive motion planning level (Reynolds 1987; Qiu and Hu 2010; Moussaïd, Perozo, Garnier, Helbing, and Theraulaz 2010). However, the ongoing communication and coordination among group members may affect how the agents value a particular action, path, and location. For a simulation of human-like crowd behavior, we must account for social interactions not just at a reactive level, but at a higher level for making route choices over time.

A dominant model of human coordinated behavior was advanced by Herbert Clark (Clark 1996; Clark and Brennan 1991). A key contribution of this paper is that we devised a computational model informed by Clark's Common Ground (CG) model that both inherits the social realism provided by the CG model and is computationally tractable for a large number of groups and individuals. In our approach, the task of navigation in a group is viewed as performing a joint activity with involved agents, which requires effective coordination among group members. Each agent's high-level choices on the route and walking strategies are affected by the group coordination result.

Since CG is a well-researched interaction model, any significant differences will provide insight concerning the effect of employing such a model into a large scale simulation may be important. For example, if we find a non-CG simulation to always 'out-perform' a CG-based model in terms of time-togoal for agents, and level of congestion, this might indicate that models that do not consider the cost of coordination may fail to capture the real crowd effects. This can be significant if we were designing a mall with certain target number and placement of shops for a target number of visitors. Such underestimations of crowd complexity may, for example, cause designers to be overly optimistic about the size of corridors or of evacuation rates in an emergency.

The remainder of this paper is organized as follows. Section 2 gives a short overview of foundational research to our work. Section 3 presents the design of our pedestrian model. The development of simulation based on the proposed pedestrian model is described in Section 4. Section 5 provides the results of simulation and performance. We draw conclusions and provide possible future research directions in Section 6.

\section{FOUNDATION}

Our model addresses both high level (route choice, activity planning) and low level (steering, reaction) navigation tasks towards the simulation of social group interaction. The former aspect is managed by incorporating Clark's CG theory into the pedestrian model, and the latter is derived using a Velocity Obstacle (VO) model (Fiorini and Shillert 1998).

\subsection{Common Ground Theory}

People engage in a joint activity when they act in coordination with others to pursue a common goal. The concept common ground is established as a mechanism by which participants involved in a joint activity coordinate their actions. It considers the mutual knowledge, beliefs, and assumptions among individuals in a collaborative process. According to Clark, $p$ is common ground for members of group $\mathscr{G}$ if and only if (Clark 1996)):

1. members of $\mathscr{G}$ know that $p$;

2. members of $\mathscr{G}$ know that members of $\mathscr{G}$ know that $p$;

3. members of $\mathscr{G}$ know that members of $\mathscr{G}$ know that members of $\mathscr{G}$ know that $p$.

Suppose that A and B are heading to the seats in a football stadium. As they pass a concession stand, A decides to get some refreshments. A informs B of his plan to visit the stand and to return to their current location, $x$. We denote the plan to split up and reunite at $x$ as P. For the plan to succeed, A needs to know that B knows the plan P. This, however, is insufficient for coordinate. B needs to know that A knows that she is privy to $\mathrm{P}$, otherwise she might think A would be left looking for her. Furthermore, if the agreement 
ends here, A may not know that B knows that he knows the plan, and may, therefore not be confident to execute the plan. Hence, A needs to know that B knows that he knows the plan.

This common ground may be communicated verbally, or may be enacted through action. In our example, A may signal his intention by pointing toward the concession stand and pointing to their current location $x$. This requires that $\mathrm{B}$ be within the range of sight and be looking at $\mathrm{A}$. A needs to see that $\mathrm{B}$ is looking at A, and has signaled agreement (e.g., by nodding). B needs to see that A sees her nodding. Finally A needs to see that B sees that he has seen and acknowledged the plan.

The CG model has two distinct strengths: (1) It is scientifically validated model on human behaviors. (2) It consists of logical components that support an explicit representation of coordination process, so lends itself to a direct implementation. Clark's CG model allows us to simulate the coordination process at a higher macro-level without actually modeling the negotiation process from the level of 'communicative intent', which coordination-naive systems need to do. This makes the simulation tractable while still maintaining realism.

The CG model has become the basis of a large volume of work in the domain of Computer-Supported Cooperative Work (CSCW) (Monk 2003; Neale, Carroll, and Rosson 2004; Convertino, Mentis, Rosson, Carroll, Slavkovic, and Ganoe 2008), AI agents (Klein, Feltovich, Bradshaw, and Woods 2005), and robotics (Kirby, Simmons, and Forlizzi 2009) for coordination-related problems.

\subsection{Velocity Obstacle Model}

A low-level reactive steering for collision-free and cohesive motion of agents are generated based on a VO model (Fiorini and Shillert 1998), using a publicly available RVO2 Library (van den Berg, Guy, Snape, Lin, and Manocha 2011). A VO of an agent $A$ is a set of all velocities that will eventually cause collisions with others, assuming that the other agents keep their current velocities. Hence, it is guaranteed that no collision occurs if $A$ chooses a velocity outside the $V O$. The $V O$ of agent $A$ induced by agent $B$ with velocity $v_{B}$ is defined as:

$$
V O_{A}^{B}\left(v_{B}\right)=\left\{v_{A} \mid \exists t>0:\left(v_{A}-v_{B}\right) t \in D\left(x_{B}-x_{A}, r_{A}+r_{B}\right)\right\}
$$

where $A$ and $B$ have position $x_{A}$ and $x_{B}$, and radius $r_{A}$ and $r_{B}$, respectively. $D(x, r)$ represents a disc of radius $r$ centered at $x$. The RVO2 Library computes velocities for each agent taking into account the reactive behavior of the other agents by implicitly assuming that the other agents draw a similar collision avoidance reasoning. Therefore, it chooses a new velocity that is the average of its current velocity and a velocity that lies outside the other agents VO (van den Berg, Lin, and Manocha 2008; Guy, Curtis, Lin, and Manocha 2012).

\section{PEDESTRIAN MODEL}

We distinguish pedestrian navigation tasks at the following three levels: global path planning, coordinated activity planning, and reactive local planning. Our focus in this paper is on modeling pedestrian behaviors at the second (Section 3.2) and third levels (Section 3.3).

\subsection{Notation and Overview}

Our model assumes that the group memberships and goal of groups are known a priori and not subject to change throughout the simulation. Goals are specific and definable geographic points in a given virtual environment. An agent with its personal identifier $j$ and group membership $i$ is represented as $A_{i j}$. The set of all members of group $i$, denoted $\mathscr{G}_{i}$, have the common initial position $p_{i}^{0}$ and final goal $g_{i}$. A leader agent $L_{i}$ is selected per group. Each agent $A_{i j}$ might insert a sub-goal $s_{i}$ while traveling with group members.

Figure 1 shows the relationship among the three navigation levels. A collision free route among static obstacles toward $g_{i}$ is precomputed for each group $i$. This produces a sequence of waypoints from $p_{i}^{0}$ to $g_{i}$. From these, a set of velocities are generated to connect the waypoints. We shall denote these coarse global-level velocities as guiding velocities to distinguish them from other velocity values in this paper. 


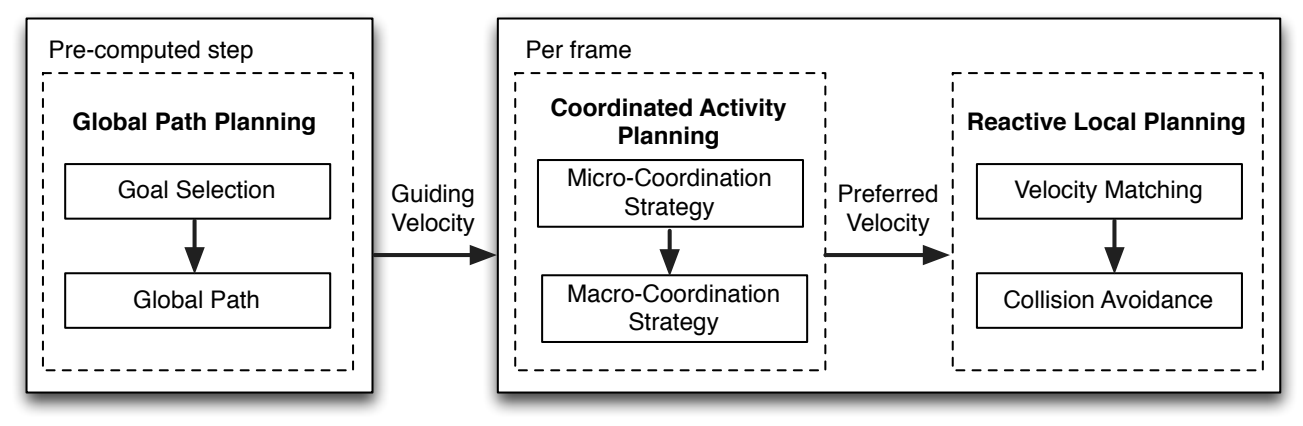

Figure 1: Three steps of planning in our pedestrian model.

Note that a global path does not specify how exactly an agent should move from one waypoint to the next one along the planned path. While adhering to the global path plan of its group from a long-term perspective, an agent determines its short-term activity plans by interacting with other group members at the coordinated activity planning level (Section 3.2). As a result, a set of preferred velocities are derived. Then an agent considers the maintenance of appropriate proximity to its group members as well as the presence of other agents and moving obstacles to determine its immediate steering in the reactive local planning step (Section 3.3).

\subsection{Group Coordination Model}

One of advantages of our model is that it can handle sub-goals that may be generated stochastically or through interaction between agents and the environment. For example, a members of groups may have to visit the restroom (stochastically generated sub-goal), or a member of a group may see a store that sells something he is interested in (sub-goal generated through interaction with the environment). This allows some agent $A_{i j}$ traveling to a final goal $g_{i}$ with $\mathscr{G}_{i}$ to insert a sub-goal $s_{i}$ (e.g., go to shoe store) that is expressed as a new sub-goal location. When a sub-goal is introduced for an agent, this necessitates the generation of activity plan for its accomplishment. We call the activity plan a sub-plan. While adjusting its activities for $s_{i}, A_{i j}$ should maintain the $\mathrm{CG}$ with the rest of members to ensure the group coordination. We introduce a Group Coordination Model (CGM) into a pedestrian architecture to address this aspect. The CGM is expressed in the form of the set:

$$
C G M:\left[\mathscr{G}_{i}, g_{i}, S P_{n}, P r_{m}\right],
$$

where $\mathscr{G}_{i}$ is the set of members of group $i, g_{i}$ is a goal of the group, $S P_{n}$ is a sub-plan, and $P r_{m}$ is a list of ordered preference probability for possible sub-plans. Note that we are not simulating the process of CG negotiation, and agents do not plan their courses of activities from 'first-principles'. Rather, group behaviors are selected at run-time from a set of pre-designed sub-plans that are grounded in CG theory.

\subsubsection{Group Coordination Sub-plans}

Sub-plans relate to sub-goals and methods to achieve group coordination. We divide the sub-plans into two parts: macro-coordination strategy (macroCS) and micro-coordination strategy (microCS). The macroCS defines a macro-level action plan. For instance, if the Divide-and-Wait macroCS is selected, $A_{i j}$ splits up from a group and pursues its sub-goal $s_{i}$ while the rest of $\mathscr{G}_{i}$ maintain their positions until $A_{i j}$ returns. The microCS defines a selection of communicative acts to activate a specific macroCS. For example, $A_{i j}$ can use a physical act (e.g., pointing at $s_{i}$ ) to express its interest of visiting $s_{i}$ and initiate the choice of macroCS. After the macroCS is selected, members of $\mathscr{G}_{i}$ will act out their microCS acknowledgement actions (e.g., nodding). Detailed descriptions of macroCS and microCS are given in Sections 3.2.2 and 3.2.3. 
We define a sub-plan $S P_{n}$ in the form of the set:

$$
S P_{n}:\left[k, r, s_{i}, \mathscr{M}_{\beta}, \mu_{\gamma}\right],
$$

where $k$ is a sub-plan identifier, $r$ is a set of roles for members of $\mathscr{G}_{i}$ in a sub-plan, $s_{i}$ is a proposed sub-goal by any member $A_{i j}, \mathscr{M}_{\beta}$ is macro-coordination strategy, and $\mu_{\gamma}$ is a micro-coordination strategy. Roles specify which agent does what with respect to a specific sub-plan. For example, $A_{i j}$ takes a role of initiator if it proposes a sub-goal, and other members become respondents to $A_{i j}$.

We follow a format of the distributed Multi-Agent Reasoning System (dMARS) specification (D'Inverno, Luck, Georgeff, Kinny, and Wooldridge 2004; Hoogendoorn and Soumokil 2010) and specify the sub-plan by means of four elements: (1) Invocation condition which defines under what conditions a plan should be considered. (2) Context of the plan which specifies the environment and situation under which the plan is executable. (3) Body of the plan which describes the course of actions using three constructs. QUERY allows an agent to check whether it currently meets a necessary condition to take a particular action. EXECUTE directs the execution of an action. ASSERT updates a status of the sub-plan. (4) Internal action which directs what should be executed in case a plan succeeds or fails.

\subsubsection{Macro-Coordination Strategy}

A macro-coordination strategy (macroCS) refers to the macro-level action plan that is adopted by a group based on the group members' preferences. Examples of macroCS for navigation are listed in Table 1. The set of macro-behavior plans are not limited to the given list. A simulator can add any kind of macro-behavior strategies if they format it for the dMARS specification.

Table 1: A list of MacroCSs.

\begin{tabular}{|l|l|l|}
\hline Name & Role & Action Description \\
\hline Lead-and-Follow & $\begin{array}{l}\text { Initiator } \\
\text { Respondent }\end{array}$ & $\begin{array}{l}\text { Leads other members towards a sub-goal } s_{i} \\
\text { Follows an initiator }\end{array}$ \\
\hline Divide-and-Proceed & $\begin{array}{l}\text { Initiator } \\
\text { Respondent }\end{array}$ & $\begin{array}{l}\text { Proceds to a sub-goal } s_{i} \text {, and } \\
\text { If the sub-goal is accomplished, proceed to a goal } g_{i} \\
\text { Proceds to a goal } g_{i}\end{array}$ \\
\hline Divide-and-Wait & $\begin{array}{l}\text { Initiator } \\
\text { Respondent }\end{array}$ & $\begin{array}{l}\text { Proceds to a sub-goal } s_{i} \text {, and } \\
\text { If the sub-goal is accomplished, returns to its group members } \\
\text { Stay and wait until an initiator agent returns }\end{array}$ \\
\hline
\end{tabular}

The general form of the sentence of a plan consists of predicates, their parameters, and a pair of action directions:

$$
<\text { predicate }>(<\text { parameters }>) \quad[x, y] .
$$

The predicate is a function over parameters. A pair $[x, y]$ specifies the resulting actions of the predicate depending on its success. Line $x$ is executed if an expression in the predicate is positive, and line $y$ is executed otherwise.

The Divide-and-Wait plan for an initiator agent $A_{i j}$ is shown in Table 2. The invocation condition signifies that an event "RecivedAckFrom $\mathscr{G}_{i}$ " should be present to activate the plan; the plan is executable only in case $A_{i j}$ received an acknowledgment for the proposal of visiting $s_{i}$ from its group members. This condition ensures that all the group members are on common ground for the new activity plan of $A_{i j}$. The process through which an agent acquires an acknowledgment is framed as a microCS, and detailed in a following section. A context describes that the plan is valid only when $A_{i j}$ is attracted by a sub-goal $s_{i}$. When these conditions are met, a series of actual physical movements can be taken. Firstly, $A_{i j}$ queries if it has arrived at the sub-goal (HasArrivedAt $s_{i}$ ). Line 9 is executed on success of (HasArrivedAt $s_{i}$ ), and line 5 is executed otherwise. If $A_{i j}$ has not reached $s_{i}$, it keeps moving towards the sub-goal (MoveTowards $s_{i}$ ). A success of activity is declared when $A_{i j}$ arrives at $s_{i}$. This invokes the execution of next step activity for $A_{i j}$ to return to its group $\mathscr{G}_{i}$ (MoveTowards $\mathscr{G}_{i}$ ). Thereafter, $A_{i j}$ is moving towards the rest of $\mathscr{G}_{i}$ and the whole plan is terminated when the group is reunited. 
Park, Quek, and Cao

Table 2: Example macroCS: Divide-and-Wait plan for $A_{i j}$ with a role initiator.

\begin{tabular}{|c|ll|l|}
\hline line no. & @ DivideAndWait & Activity Name \\
\hline 1 & RecivedAckFrom $\mathscr{G}_{i}$ & & Invocation condition \\
2 & AttractedBy $s_{i}$ & Context \\
& & \\
4 & QUERY HasArrivedAt $s_{i}$ & {$[9,5]$} & Plan body \\
5 & EXECUTE MoveTowards $s_{i}$ & {$[4]$} & \\
6 & QUERY isReunitedWith $\mathscr{G}_{i}$ & {$[10,7]$} & \\
7 & EXECUTE MoveTowards $\mathscr{G}_{i}$ & {$[6]$} & \\
& & & \\
9 & ASSERT ArrivedAt $s_{i}$ & {$[6]$} & Internal action for success \\
10 & ASSERT Reunited & {$[11]$} & \\
\hline
\end{tabular}

\subsubsection{Micro-Coordination Strategy}

A micro-coordination strategy (microCS) defines a set of communicative actions that simulate how group members get each other to understand what they intend, and initiate a particular macroCS. The kind of information and communicative acts exchanged among agents, and the physical configuration that permits the communication to function are included in a microCS definition. In our model, a range of microCSs may be defined with different environment conditions. For example, different kinds of non-verbal microCSs may support visual coordination using gestures. In a sparse crowd, longer-range gestures may be used with greater tolerance for gaze-direction specificity for CG maintenance. In a denser crowd, the microCS initiator may first have to gain attention verbally (speech coordination) before employing gestures. In a very dense crowd, agents may have to employ speech-only coordination, requiring that the communicating agents be much closer together.

The general form of the body of a microCS, $\mu_{\gamma}$, is described in Table 3. This action body may be activated, for example, if $A_{i j}$ is within some range $d^{\prime}$ from the respondent agent, where $d^{\prime}$ is greater than distance $d$ that is necessary for the success of signaling action $\mathscr{S}$. In this case, line 1 of the action plan is for $A_{i j}$ to move within distance $d$ of the respondent before performing $\mathscr{S}$ in line 2 . If $A_{i j}$ is further than $d^{\prime}$ from the respondent, then $\mu_{\gamma}$ will not apply.

Table 3: Body of a microCS.

\begin{tabular}{|c|l|}
\hline line no. & Action Specification \\
\hline 1 & $A_{i j}$ acts to satisfy conditions (physical configurations) for $\mu_{\gamma}$ \\
2 & $A_{i j}$ performs the signaling action, $\mathscr{S}$ \\
3 & Select the appropriate macroCS $\mathscr{M}_{\beta}$ \\
4 & $A_{i k}, k \neq j \in \mathscr{G}_{i}$ signifies acknowledgement for $\mu_{\gamma}$ \\
\hline
\end{tabular}

Table 4: Example microCS: Visual coordination.

\begin{tabular}{|c|ll|l|}
\hline line no. & @ GetVisualCoordination & Activity Name \\
\hline 1 & AttractedBy $s_{i}$ AND IsWithinDistance $\left(d^{\prime}\right)$ of $L_{i}$ & $\begin{array}{l}\text { Invocation condition } \\
\text { Context }\end{array}$ \\
2 & TravelWith $\mathscr{G}_{i}$ & {$[7,5]$} & Plan body \\
& & {$[4]$} & \\
5 & QUERY IsWithinDistance $d$ & {$[9,10]$} & \\
6 & EXECUTE MoveTowards $L_{i}$ & {$[6]$} & \\
7 & QUERY RecivedAckFrom $L_{i}$ & & \\
& EXECUTE ProposeMacroPlan $\mathscr{M}_{\beta}$ & {$[11]$} & Internal action for success \\
9 & ASSERT ActivateMacroPlan $\mathscr{M}_{\beta}$ & {$[11]$} & Internal action for fail \\
10 & ASSERT FailToGetAcknowledgment & & \\
\hline
\end{tabular}

An example of visual coordination is shown in Table 4. When $A_{i j}$ is attracted by $s_{i}$ and within some range $d^{\prime}$ from the group members while traveling together, it launches the visual coordination mode. If 
$A_{i j}$ and the rest of $\mathscr{G}_{i}$ are within a given distance range (IsWithinDistance $d$ ), $A_{i j}$ suggests a particular macroCS $\mathscr{M}_{\beta}$ to achieve $s_{i}$ (ProposeMacroPlan $\mathscr{M}_{\beta}$ ). The macroCS $\mathscr{M}_{\beta}$ is selected as considering the group preference $P r_{m}$. If the distance constraint is not met, $A_{i j}$ approaches the group until they are close enough so that $A_{i j}$ can retry the proposal of using $\mathscr{M}_{\beta}$. If group members give acknowledgments for the proposal, $\mathscr{M}_{\beta}$ is activated and group members perform a sequence of activities described in $\mathscr{M}_{\beta}$. An indication of acknowledgment could be a head nod in a visual coordination mode. Not receiving a signal for an acknowledgment means that $A_{i j}$ failed to get coordinated with its group members for pursuing $s_{i}$. A failure of activity is declared, and then $A_{i j}$ may suggest other available microCS considering the situation.

\subsection{Reactive Local Planning Model}

VO models do not provide support for group-based movement. In order to simulate a cohesive movement pattern among group members such as walking together, we incorporate the velocity matching into members. We use a group leader's forward velocity vector $V_{\text {leader }}$ as a base for the velocity matching. For each group member $A_{i j}$, a distance to leader $L_{i}$ projected on $V_{l e a d e r}$ is computed:

$$
V_{\text {leader }} \cdot \operatorname{distance}\left(L_{i}, A_{i j}\right) \text {. }
$$

A distance greater than a higher threshold (1.5m in our simulation) implicates $A_{i j}$ is far behind $L_{i}$, therefore $A_{i j}$ accelerates while $L_{i}$ decelerates. A distance less than a lower threshold (-1.5m in our simulation) means $A_{i j}$ is far ahead $L_{i}$, therefore $L_{i}$ accelerates and $A_{i j}$ decelerates.

\section{DEVELOPMENT OF PEDESTRIAN SIMULATION}

In this section we discuss the development of crowd simulation framework based on our pedestrian model.

\subsection{Environment Model}

In our system, a virtual simulation space containing interest points is represented as a roadmap in which overall connectivity information is represented as a graph. Interest points serve as potential sub-goals to agents. Given the initial position, $p_{i}^{0}$, and final goal, $g_{i}$, of group $i$, the $\mathrm{A}^{*}$ algorithm is used to generate a global path. Any type of global path planners can replace the use of $A^{*}$ algorithm as long as it generates a collision-free route around static obstacles.

\subsection{Sub-goal Selection}

We classify sub-goals into two categories. The first type are stochastically generated sub-goals, and agents are triggered to visit in this type of sub-goals using a random event generator. An example of this type of sub-goal is a restroom visit. The second type is a sub-goal generated through interaction with the environment. Let the set of possible sub-goal destinations be $W_{\alpha}, \alpha=1, \cdot \cdot, K$. Each agent would have a vector of interests $I_{j, \alpha}, \alpha=1, \cdot \cdot K$, denoting its interest in $W_{\alpha}$. Interest values range from 0 to 1.0. If $A_{i j}$ passes within a pre-specified distance from some $W_{\alpha}$, and if $I_{j, \alpha}$ exceeds some likelihood-of-visit threshold $L H_{j}, W_{\alpha}$ would be selected as a potential sub-goal. The value of $L H_{j}$ ranges from 0 to 1.0 , and is generated randomly per agent. If a $W_{\alpha}$ is selected, $A_{i j}$ initiates a microCS to introduce it as a sub-goal to the group members. In a shopping-mall scenario, $W_{\alpha}, \alpha=1, \cdot \cdot K$ would be a list of $K$ shops in the mall.

\subsection{Sub-plan Selection}

Two kinds of sub-plan selection are needed for microCS and macroCS, respectively. The selection of some microCS $\mu_{i}$ could be dependent on the state of the environment, and the state of the agents. For example, a visual gestural microCS requires that the recipient agent must be within some range of, and looking at the signaling agent. If more than one $\mu_{i}$ fits the conditions, one is selected randomly. As shown in Tables 3 and 4 , a body of each $\mu_{i}$ further specifies a set of actions to be performed by the agents. Some of these 
actions may actually help to satisfy the trigger conditions of a microCS. For example, the requirement that the recipient agent be looking at the signaling agent may be satisfied by having the signaling agent move within the field of view of the recipient.

An initiator agent $A_{i j}$ suggests a selection of $\mathscr{M}_{\beta}$ and should receive acknowledgement of the choice by other members of $\mathscr{G}_{i}$. Given a potential sub-goal $W_{\alpha}$, an average interest $I_{i, \alpha}$ in $W_{\alpha}$ of group members is used to make a decision:

$$
I_{i, \alpha}=\frac{\sum_{k\left(\neq j, k \in \mathscr{G}_{i}\right)} I_{k, \alpha}}{n-1}
$$

where $n$ is the number of members of group $i$. Using the list of $\operatorname{Pr}_{m}$, a particular region where the $I_{i, \alpha}$ value falls is determined, and a corresponding $\mathscr{M}_{\beta}$ to the region is selected.

\section{EXPERIMENT AND RESULTS}

We conducted a series of studies to show the qualitative and quantitative differences in simulation results with and without incorporation of the Common Ground model. We use three simulation configurations and compare the arrival rate at a designated destination and congestion levels at key points to show the impact of coordinated groups in the simulation. Six-hundred agents in total are organized as (1) 600 of solely individual agents, (2) 150 simple groups of 4 members without considering the CG model, and (3) 150 groups of 4 members with incorporating the CG model. We shall call each of the three configurations CI (for individuals), CnCG (non-CG), and CCG (CG model), respectively. All the results in this section have been averaged over 10 independent simulations.

\subsection{Scenario}

A shopping mall scenario has been designed for the experiments. Our shopping mall model contains 4 restrooms (green squares labeled as R1, R2, R3, and R4) and 9 shops (orange squares) as potential sub-goals as shown in Figure 2. Each cell in the 2D grid denotes a $5 \mathrm{~m} \times 5 \mathrm{~m}$ area. For all the individual agents in CI and agents of groups in $\mathrm{CnCG}$ and $\mathrm{CCG}$, an exit $E$ is given as a final goal. Initial positions of agents are randomly distributed in a shopping mall, taking care that members within groups are collocated. Though agents in CI are all individuals, we initialized the simulation with agents clustered in groups of 4 to have the same initial conditions as $\mathrm{CnCG}$ and CCG. Starting from initial positions, agents walk around the shopping mall and eventually proceed to the exit. A random event generator triggers agents to visit a nearby restroom. In case no nearby restroom is within a distance range $(25 \mathrm{~m} \times 25 \mathrm{~m}$ in our implementation), they do not take any special actions and resume their original navigation plans. As agents pass by shops, they might be attracted to some shop within a range $(15 \mathrm{~m} \times 15 \mathrm{~m}$ in our implementation).

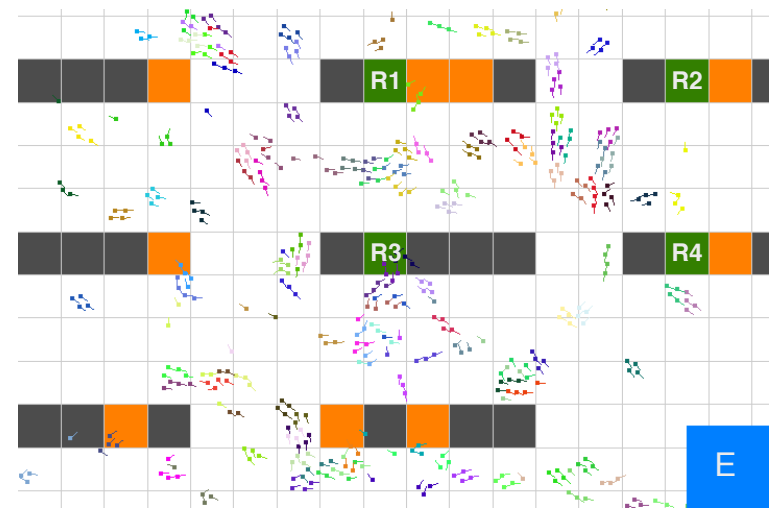

Figure 2: A snapshot of the shopping mall. 


\subsection{Simulation Parameter Choices}

For our studies, we implemented two microCS models: a visual and a verbal coordination model. For simplicity, we specify a distance among agents as the only trigger condition for these microCSs. Specifically, the verbal coordination mode requires the agents must be within a short distance (a distance constraint $d_{u}^{\prime}$ is set to $3 \mathrm{~m})$, whereas the visual coordination mode is effective in a larger range of area $\left(d_{v}^{\prime}=5 \mathrm{~m}\right)$. When the agents are within the range of $d_{u}^{\prime}$, the choice of microCS is made randomly because both conditions, $d_{u}^{\prime}$ and $d_{v}^{\prime}$, are satisfied. If none of distance constraints is met, an initiator agent approaches to the respondents and retry the microCS up to three times.

We use two values of $\operatorname{Pr}_{m}\left(P_{1}=0.4\right.$ and $\left.P_{2}=0.7\right)$ to select among the three macroCSs defined in Table 1 (i.e., $\mathscr{M}_{1}$ for $I_{i, \alpha}<P_{1}, \mathscr{M}_{2}$ for $P_{1}<I_{i, \alpha}<P_{2}, \mathscr{M}_{3}$ for $I_{i, \alpha}>P_{2}$ ). $\mathscr{M}_{1}, \mathscr{M}_{2}$, and $\mathscr{M}_{3}$ represent the Divideand-Proceed, the Divide-and-Wait, and the Lead-and-Follow macroCSs respectively. To simulate simple groups in $\mathrm{CnCG}$, we set group members to always follow an initiator agent of a sub-goal (i.e., all group members will satisfy all sub-goals together, before proceeding to the final goal).

\subsection{Arrival Rate}

Figure 3(a) shows a comparison of arrival rates among the three configurations. The $\mathrm{x}$-axis represents time steps and the y-axis is the percentage of agents who have arrived at the exit $E$. This percentage will increase over time and the slope of the line signifies the current rate at which agents reached an exit. For example, after $2.2 k$ time steps we see that all the agents have arrived at the exit in CI, whereas $92 \%$ and 96\% of agents have reached in CnCG and CCG, respectively. At the beginning of the simulation up to 400 time steps, a similar increases in the arrival rate are observed in all of the three settings, as those agents who have been located relatively close to an exit arrive at the exit quickly.

For agents in a group, either CnCG or CCG, the arrival rates are slower than that of CI. This is due to a multitude of factors. In CnCG and CCG, group members maintain group cohesion by velocity matching, and result in taking longer time to complete the travel. For example, if some of the members in a group are stuck in a congestion, the rest of members slow down their speeds to walk with them. Another factor affecting the arrival rate in our coordinated group model (CCG) is the use of Divide-and-Proceed strategy in macroCSs. The strategy allows some member agents to proceed towards an exit while their member with a sub-goal is visiting the place, hence results in faster arrivals at the exit.

Simulations on individual agents (CI) and simple groups (CnCG) yield best- and worst-case scenarios respectively and suggest good first-order approximations. However, the simulation with our group coordinated model captures a more realistic group navigation pattern.

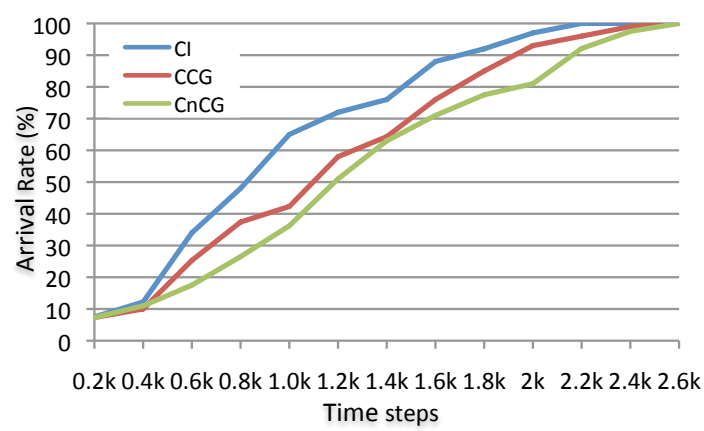

(a)

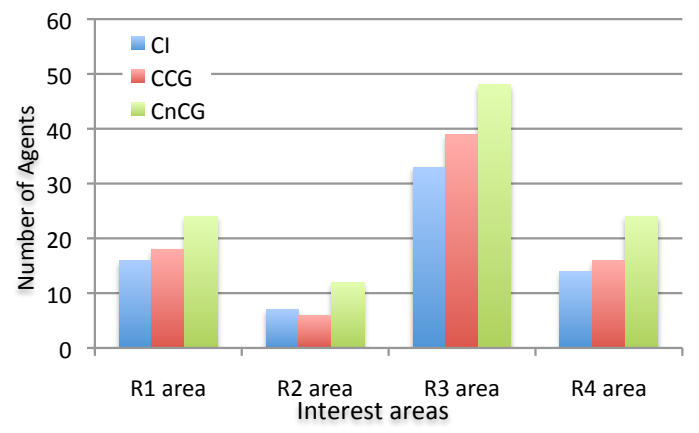

(b)

Figure 3: (a) Arrival rates of CI, CnCG and CCG. (b) Congestion levels measured as the number of agents in unit areas of $\mathrm{CI}, \mathrm{CnCG}$, and CCG. 


\subsection{Congestion Level}

In order to compare the congestion levels, we examined the accumulated number of agents for 200 time steps which have been in $15 \times 15 \mathrm{~m}^{2}$ ranges centered at the stochastic sub-goal locations (R1, R2, R3, and R4). Figure 3(b) shows the congestion levels at the four areas from 600 to 799 time steps in CI, CnCG, and CCG simulations. Comparing to R2 and R4, R1 and R3 are located along the main path towards an exit $E$, and higher congestion levels in those areas are observed in all the three configurations. Since simple group members in $\mathrm{CnCG}$ always follow an initiator and visit a sub-goal, the highest degree of congestion is observed.

We see more local congestion in CCG than CI. For agents of CCG, the coordination activities to establish and maintain common ground incur costs at the level of entire simulation. For example, a pathway is blocked by some initiator agents who are retrying the micro-coordination with group members at a failure of receiving an acknowledgment. An execution of some macro-level actions also adds the cost; when executing the Divide-and-Wait plan, those respondent agents holding their positions might cause a congestion.

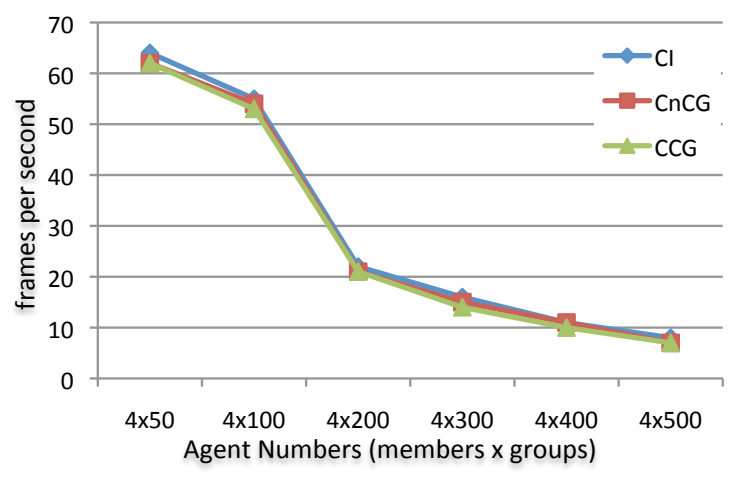

Figure 4: Frames per second measured in the CI, CnCG and CCG configurations.

\subsection{Scalability}

We evaluated the scalability of our approach with respect to the number of groups and total agents. The simulation was tested on a desktop with an Intel i7 $3.20 \mathrm{GHz}$ CPU, and 4GB system memory. Frames per second are measured in the CI, CnCG and CCG configurations. As shown in Figure 4, the computation overhead for group coordination simulation in CCG is not significant. Taking $15 \mathrm{fps}$ as the minimum interactive rate, the simulation with our group coordination model runs at an interactive rate up to 1,200 of agents of 300 groups.

\section{CONCLUSION}

In this paper, we presented a pedestrian model founded on the well-researched CG Theory to incorporate the impact of social interaction among group members in the crowd simulation. In particular, consideration of how each agent evaluates a particular action, path, and location depends on the ongoing communication and coordination among group members allowed us to reproduce realistic group dynamics in a pedestrian simulation. A series of experiment results showed the qualitative and quantitative differences in simulation results with and without incorporation of the CG model. In addition, our model is computationally efficient in that the negotiation process from the cognitive and reasoning levels of agents are not necessary to simulate the coordinated group behaviors. A set of performance tests demonstrated that our approach does not introduce a significant computational overhead into a simulation. 
Park, Quek, and Cao

Our future research direction is in extending the micro-coordination specifications towards further sophistication. In our current model, we only consider a distance between initiator and respondent agents to trigger a specific micro-coordination strategy. Employing the state of the environment conditions including the level of crowdedness, and the state of agents including the gaze direction and body orientation as trigger conditions for micro-coordination selection will bring more realistic pedestrian dynamics and provide a valuable insight concerning the effect of social interaction and coordination in a pedestrian simulation.

\section{ACKNOWLEDGMENTS}

This research has been partially funded by NSF grant, "EAGER: Drummer Game: A Massive Interactive Socially-Enabled Strategy Game," IIS-0940723, and “CRI: Interfaces for the embodied mind,” IIS-0551610.

\section{REFERENCES}

Antonini, G., M. Bierlaire, and M. Weber. 2006. "Discrete choice models of pedestrian walking behavior". Transportation Research Part B: Methodological 40 (8): 667 - 687.

Clark, H. H. 1996, May. Using Language. Cambridge University Press.

Clark, H. H., and S. E. Brennan. 1991. "Grounding in communication”. In Perspectives on Socially Shared Cognition, edited by L. B. Resnick, J. M. Levine, and S. D. Teasley, 127-149. Washington DC: American Psychological Association.

Coleman, J. S., and J. James. 1961. "The Equilibrium Size Distribution of Freely-Forming Groups". Sociometry 24 (1): pp. 36-45.

Convertino, G., H. M. Mentis, M. B. Rosson, J. M. Carroll, A. Slavkovic, and C. H. Ganoe. 2008. "Articulating common ground in cooperative work: content and process". In Proceeding of the twentysixth annual SIGCHI conference on Human factors in computing systems, edited by M. Czerwinski, A. M. Lund, and D. S. Tan, CHI '08, 1637-1646. New York, NY, USA: ACM.

D'Inverno, M., M. Luck, M. Georgeff, D. Kinny, and M. Wooldridge. 2004. "The dMARS Architecture: A Specification of the Distributed Multi-Agent Reasoning System”. Autonomous Agents and Multi-Agent Systems 9 (1-2): 5-53.

Endrass, B., E. André, M. Rehm, A. A. Lipi, and Y. Nakano. 2011. "Culture-related differences in aspects of behavior for virtual characters across Germany and Japan". In The 10th Intl. Conf. on Autonomous Agents and Multiagent Systems, edited by K. Tumer, P. Yolum, L. Sonenberg, and P. Stone, Volume 2 of AAMAS '11, 441-448. Richland, SC: International Foundation for Autonomous Agents and Multiagent Systems.

Fiorini, P., and Z. Shillert. 1998. "Motion Planning in Dynamic Environments using Velocity Obstacles". International Journal of Robotics Research 17:760-772.

Guy, S. J., S. Curtis, M. C. Lin, and D. Manocha. 2012, January. "Least-effort trajectories lead to emergent crowd behaviors". Phys. Rev. E 85:016110.

Hoogendoorn, M., and J. Soumokil. 2010. "Evaluation of virtual agents utilizing theory of mind in a real time action game". In Proceedings of the 9th Intl. Conf. on Autonomous Agents and Multiagent Systems, edited by van der Hoek, Kaminka, Lespérance, Luck, and Sen, Volume 1 of AAMAS '10, 59-66. Richland, SC: International Foundation for Autonomous Agents and Multiagent Systems.

Hoogendoorn, S. P., and P. H. L. Bovy. 2004. "Pedestrian route-choice and activity scheduling theory and models". Transportation Research Part B: Methodological 38 (2): 169 - 190.

Hughes, R. L. 2002. "A continuum theory for the flow of pedestrians". Transportation Research Part B: Methodological 36 (6): 507 - 535.

James, J. 1953. "The Distribution of Free-Forming Small Group Size”. American Sociological Review 18 (5): 569-570. 


\section{Park, Quek, and Cao}

Kirby, R., R. Simmons, and J. Forlizzi. 2009, 27 2009-oct. 2. "COMPANION: A Constraint-Optimizing Method for Person-Acceptable Navigation". In Robot and Human Interactive Communication, 2009. RO-MAN 2009. The 18th IEEE International Symposium on, 607 -612.

Klein, G., P. J. Feltovich, J. M. Bradshaw, and D. D. Woods. 2005. "Common Ground and Coordination in Joint Activity". In Organizational Simulation, edited by W. B. Rouse and K. R. Boff, 139-184. John Wiley and Sons, Inc.

Monk, A. 2003. "Common Ground in Electronically Mediated Communication: Clark's Theory of Language Use". In HCI Models, Theories and Frameworks: Toward a Multi-Disciplinary Science, edited by J. Carroll, Chapter 10, 265-290. Morgan Kaufmann.

Moussaïd, M., N. Perozo, S. Garnier, D. Helbing, and G. Theraulaz. 2010, April. "The Walking Behaviour of Pedestrian Social Groups and Its Impact on Crowd Dynamics". PLoS ONE 5 (4): e10047+.

Narain, R., A. Golas, S. Curtis, and M. C. Lin. 2009, December. "Aggregate dynamics for dense crowd simulation". ACM Trans. Graph. 28:122:1-122:8.

Neale, D. C., J. M. Carroll, and M. B. Rosson. 2004. "Evaluating computer-supported cooperative work: models and frameworks". In Proc. of the 2004 ACM conf. on Computer supported cooperative work, edited by J. D. Herbsleb and G. M. Olson, CSCW '04, 112-121. New York, NY, USA: ACM.

Qiu, F., and X. Hu. 2010. "Modeling group structures in pedestrian crowd simulation". Simulation Modelling Practice and Theory 18 (2): 190 - 205.

Rao, A. S., and M. P. Georgeff. 1995. "BDI-agents: from theory to practice". In Proceedings of the First Intl. Conference on Multiagent Systems, edited by V. R. Lesser and L. Gasser, 312-319. San Francisco: The MIT Press.

Reynolds, C. W. 1987, August. "Flocks, herds and schools: A distributed behavioral model". SIGGRAPH Comput. Graph. 21:25-34.

Jur van den Berg and Stephen J. Guy and Jamie Snape and Ming C. Lin and Dinesh Manocha 2011. "RVO2 Library: Reciprocal Collision Avoidance for Real-Time Multi-Agent Simulation”. http://gamma.cs.unc. edu/RVO2/.

van den Berg, J., M. Lin, and D. Manocha. 2008, May. "Reciprocal Velocity Obstacles for real-time multi-agent navigation". In Robotics and Automation, 2008. ICRA 2008. IEEE Intl. Conf. on, 1928 -1935: IEEE.

\section{AUTHOR BIOGRAPHIES}

SEUNG IN PARK is a PhD candidate of Computer Science at Virginia Polytechnic Institute and State University. She received her B.S. and M.S. degrees in computer science and engineering from Ewha Womans University in South Korea, in 2003 and 2006, respectively. She is currently working on developing a crowd model for generating realistic behaviors of pedestrians and group dynamics. Her email address is spark80@vt.edu.

FRANCIS QUEK a professor of computer science and mathematics at Virginia Polytechnic Institute and State University. Francis received both his B.S.E. summa cum laude (1984) and M.S.E. (1984) in electrical engineering from the University of Michigan. He completed his Ph.D. in Computer Science at the same university in 1990. Francis is a member of the IEEE and ACM. He leads several multiple-disciplinary research efforts to understand the communicative realities of multimodal interaction, and in embodied interaction. He can be reached at quek@vt.edu.

YONG CAO is an assistant professor of computer science at Virginia Polytechnic Institute and State University. Yong received his Ph.D. degree in computer science from University of California, Los Angeles in 2005. His research interests are in crowd simulation, data-intensive visualization, and hybrid computing on many-core and multi-core systems. He also serves in the editorial board of ACM Computer in Entertainment. He can be reached at yongcao@vt.edu. 